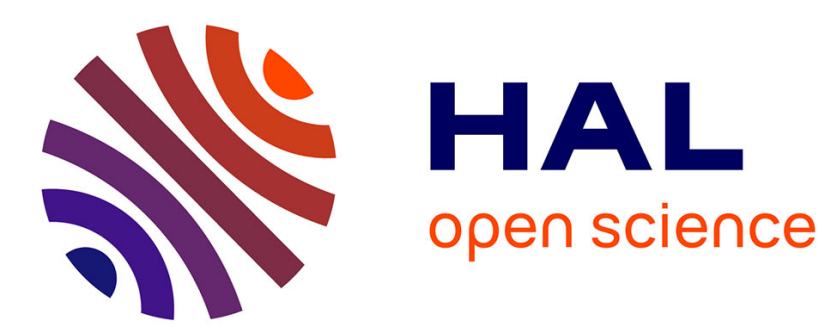

\title{
Analytical modeling of vibrations in a damaged beam using Green-Volterra formalism
}

Damien Bouvier, Nazih Mechbal, Marc Rebillat

\section{To cite this version:}

Damien Bouvier, Nazih Mechbal, Marc Rebillat. Analytical modeling of vibrations in a damaged beam using Green-Volterra formalism. European Workshop on Structural Health Monitoring 2020, Jul 2020, Palerme, Italy. pp.1-15. hal-03024158

\section{HAL Id: hal-03024158 https://hal.science/hal-03024158}

Submitted on 25 Nov 2020

HAL is a multi-disciplinary open access archive for the deposit and dissemination of scientific research documents, whether they are published or not. The documents may come from teaching and research institutions in France or abroad, or from public or private research centers.
L'archive ouverte pluridisciplinaire HAL, est destinée au dépôt et à la diffusion de documents scientifiques de niveau recherche, publiés ou non, émanant des établissements d'enseignement et de recherche français ou étrangers, des laboratoires publics ou privés. 


\title{
Analytical modeling of vibrations in a damaged beam using Green-Volterra formalism
}

\author{
Damien Bouvier, Nazih MechBAL ${ }^{[0000-0002-8928-1181]}$, and Marc \\ RÉBILLAT $^{[0000-0003-0469-8437]}$ \\ Arts et Metiers Institute of Technology, CNRS, CNAM, PIMM, HESAM Univesité, \\ F-75013 Paris, France \\ damien.bouvier@ensam.eu
}

\begin{abstract}
Structural Health Monitoring of aeronautic composite structures through Lamb waves can advantageously exploit the fact that Lamb wave damage interaction is nonlinear. However, one difficulty in this context is to be able to distinguish between nonlinearities due to the propagation (i.e. material or geometrical nonlinearities) and those due to the damage itself that are of main interest here. This work proposes to use the Green-Volterra formalism to build up a model for Lamb Wave propagation and damage interaction that is complex enough to represent both types of nonlinearities, and simple enough to be used for simulation and estimation purposes. This approach is presented for the low frequency S0 mode nonlinear propagation in a damaged beam. An analytical model of the nonlinear wave propagation is first derived, where the damage is represented with a polynomial stiffness characteristic acting via boundary conditions. This model is then used to derive the Green-Volterra series describing the nonlinear input-output relationship of the system. A modal decomposition of the Green-Volterra series is also provided. Simulations are presented, and the proposed approach is successfully compared to state-of-the-art methods based on finite-elements models.
\end{abstract}

Keywords: Lamb Waves · Nonlinear Damage · Nonlinear Propagation in composite materials · Green-Volterra model · S0 mode.

\section{Introduction and problem statement}

Structural Health Monitoring (SHM) combines advanced sensor technology with intelligent algorithms to interrogate the structural "health" condition. Generally, a SHM process entails establishing: (1) the existence of damage, (2) the damage locations, (3) the types of damage, and (4) the damage severity [1]. On the basis of the assumption that in many cases damage causes a structure to exhibit nonlinear dynamical response and that the damage monitoring process can be significantly enhanced if one takes advantage of these nonlinear effects [2], we aim here at providing 
a framework with a richer representation of nonlinear damages and nonlinear Lamb waves propagation in composite aeronautic complex structures. Such approaches in that direction have already been achieved $[3,4]$ but were not general enough in terms of damage models and did not include nonlinear Lamb wave propagation thus motivating the present study.

In order to model nonlinearities, this paper relies on the Volterra formalism. Volterra series is a model representation that describes the output signal of a system as an homogeneous series with respect to the input [5]. This approach, similar to Taylor series approximations for functions, has been shown to be an universal approximator for any nonlinear dynamical system with fading memory [6]. To correctly take into account the spatial dependency of the problem under study, this paper uses the Green-Volterra series $[7,8]$, which are an extension of the Volterra formalism incorporating the notion of Green's function. Furthermore, we use the method presented in [8] which allow to easily compute the Green-Volterra kernels for an inhomogeneous nonlinear partial differential equation where the nonlinearities are in a polynomial form.

In this paper, we thus use the Green-Volterra formalism to find analytical solutions for the S0 mode nonlinear propagation and nonlinear damage interaction in a damaged beam. Firstly, the wave propagation model used for the damaged beam is presented, where the damage is represented with a polynomial stiffness characteritic acting via boundary conditions. This model is then used to derive the Green-Volterra series describing the input-output relationship of the system. A modal decomposition of the Green-Volterra series is then given. Simulations are presented, and the proposed approach is sucessfully compared to a state-of-the-art method based on finite elements.

\section{Modelisation of the damaged beam}

\section{$2.1 \quad$ Assumptions}

In this paper, we consider a beam of length $L$ and section $S$, with fixed boundary conditions at both ends ${ }^{1}$ and a damage localized at $x=d$ (see Fig. 1). The material is homogeneous, isotrope and dissipative, with Young modulus $E$, volumic mass $\rho$ and damping factor $\gamma$; physical nonlinearity in the propagation is also taken into account, with $\epsilon$ and $\beta$ respectively the quadratic and cubic nonlinearity coefficients [9].

In order to represent the damage, we will consider that the beam is divided into two sub-beams linked by a nonlinear spring (see Fig. 2), which is characterized by a

${ }^{1}$ The presented approach is also valid for other types of boundary conditions, but for sake of clarity and concision we restrict here the presentation to this case only. 


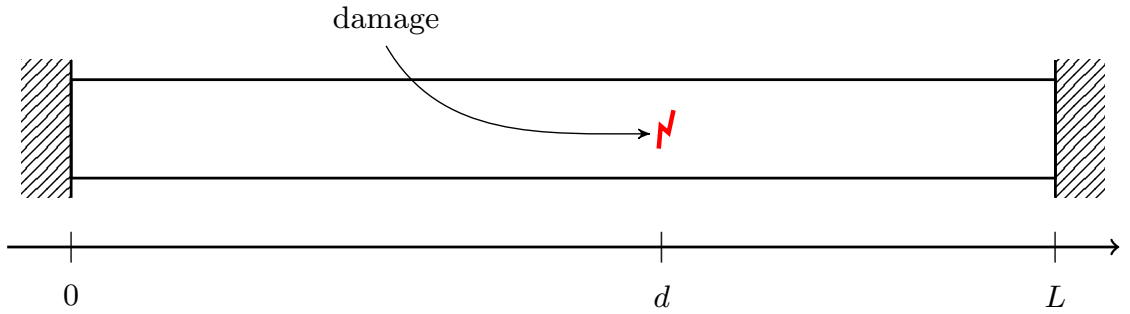

Fig. 1: Damaged beam under study.

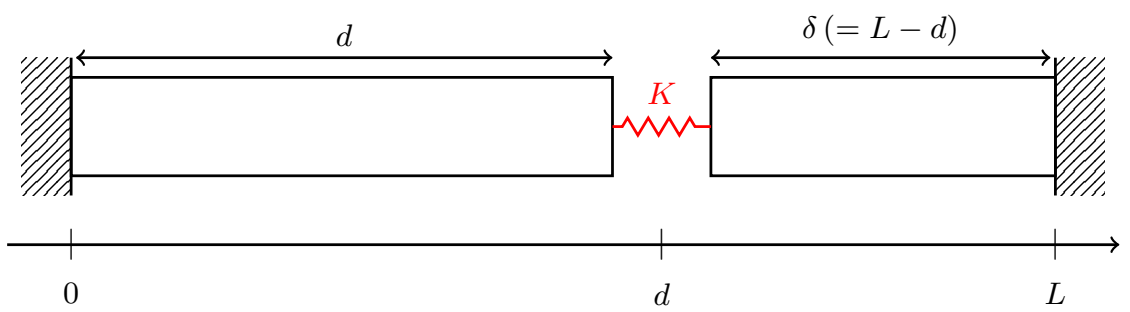

Fig. 2: Simplified model used for approximating a damaged beam.

polynomial stiffness relation, i.e.

$$
K[x]=\sum_{n=1}^{+\infty} K_{n} x^{n},
$$

with $K[x]$ the force applied by the spring at its extremities in response to an elongation $x$. We will respectively note $\Omega_{l}=\left[0, d\left[\right.\right.$ and $\left.\left.\Omega_{r}=\right] d, L\right]$ the left-part and right-part domains.

\subsection{Constitutive equations}

We consider that the beam is excited by an external force $f(x, t)$. Then, given the previous assumptions, the longitudinal vibration $u(x, t)$ follows the wave equation

$\rho S \partial_{t}^{2} u(x, t)+\gamma \partial_{t} u(x, t)-E S\left(1-\epsilon \partial_{x} u(x, t)+\beta\left(\partial_{x} u(x, t)\right)^{2}\right) \partial_{x}^{2} u(x, t)=f(x, t)$ 
for $x \in \Omega_{l} \cup \Omega_{r}$. Furthermore, the fixed extremities at $x=0$ and $x=L$ give the boundary conditions

$$
\begin{gathered}
u(0, t)=0, \\
u(L, t)=0,
\end{gathered}
$$

and the damage imposes at $x=d^{-}$and $x=d^{+}$

$$
\begin{aligned}
E S \partial_{x} u\left(d^{-}, t\right) & =K\left[u\left(d^{+}, t\right)-u\left(d^{-}, t\right)\right], \\
-E S \partial_{x} u\left(d^{+}, t\right) & =-K\left[u\left(d^{+}, t\right)-u\left(d^{-}, t\right)\right] .
\end{aligned}
$$

The constitutive equations of the damaged beam model are thus given by

$$
\Sigma:\left\{\begin{array}{l}
\begin{array}{l}
\left(1-\epsilon \partial_{x} u(x, t)+\beta\left(\partial_{x} u(x, t)\right)^{2}\right) \partial_{x}^{2} u(x, t) \\
\quad-\frac{1}{c_{L}^{2}} \partial_{t}^{2} u(x, t)-\frac{\gamma}{F_{0}} \partial_{t} u(x, t)=\frac{1}{F_{0}} f(x, t)
\end{array} \\
u(0, t)=0 \\
u(L, t)=0 \\
\partial_{x} u\left(d^{-}, t\right)=\partial_{x} u\left(d^{+}, t\right)=\partial_{x} u(d, t) \\
F_{0} \partial_{x} u(d, t)=K\left[u\left(d^{+}, t\right)-u\left(d^{-}, t\right)\right]
\end{array}\right.
$$

with $c_{L}=\sqrt{\frac{E}{\rho}}$ the longitudinal wave celerity, and $F_{0}=E S$ a factor homogeneous to a force.

The vibration $u$ is thus solution of a non-homogeneous differential equations system made up of one nonlinear propagation equation on $\Omega_{l} \cup \Omega_{r}$, two homogeneous Dirichlet boundary conditions at $x=0$ and $x=L$, one homogeneous Cauchy boundary conditions at $x=d$ and one inhomogeneous Robin boundary conditions at $x=d$. The following section will present an analytical solution of this problem using the Green-Volterra formalism.

\section{Analytical solution via Green-Volterra series}

\subsection{Volterra and Green-Volterra series}

The Volterra series [5], which have been used for many decades to model a variety of nonlinear dynamical systems, rely on the assumption that the output signal can be described as an homogeneous series, i.e. that

$$
u=\sum_{n=1}^{+\infty} u_{n}
$$


where each order $u_{n}$ is homogeneous of order $n$ w.r.t. the input force $f$, i.e. $u_{n} \propto f^{n}$. This idea allows to extend the idea of linear filter to each order of nonlinearity via the introduction of Volterra kernels. One important property of Volterra series is that it can approximate any nonlinear dynamical system with fading memory [6].

An extension of the Volterra formalism, the Green-Volterra series, has been introduced in $[7,8]$ to model problems that were also space-dependent. In this model, each homogeneous order of the output vibration $u_{n}$ is written as a convolution between a

space-time Green-Volterra kernel $g_{n}$ and multiple product of delayed versions of the input force $f$, i.e.

$$
\begin{aligned}
u_{n}(x, t)=\int_{0}^{L} \cdots \int_{0}^{L} \int_{0}^{+\infty} \cdots \int_{0}^{+\infty} g_{n}\left(x ; \xi_{1}, \ldots, \xi_{n} ; \tau_{1}, \ldots, \tau_{n}\right) \times \\
f_{n}\left(\xi_{1}, t-\tau_{1}\right) \ldots f_{n}\left(\xi_{n}, t-\tau_{n}\right) d \xi_{1} \ldots d \xi_{n} d \tau_{1} \ldots d \tau_{n}
\end{aligned}
$$

Furthermore, it has been shown in [8] how to compute the kernels $g_{n}$ from an inhomogeneous differential equation where the nonlinearities are in a polynomial form, and that it is only needed to find the Green kernel corresponding to the linear part of the differential operator (and respecting the boundary conditions of the problem). In the following, we will use this approach to derive analytical solutions for the problem stated in Eq. (7).

\subsection{Problem reformulation}

Incorporating (8) into the model (7), and sorting terms by their homogeneity order gives the sub-model that each order $u_{n}$ must follow, i.e.

$$
\Sigma_{n}:\left\{\begin{array}{l}
\partial_{x}^{2} u_{n}(x, t)-\frac{1}{c_{L}^{2}} \partial_{t}^{2} u_{n}(x, t)-\frac{\gamma}{F_{0}} \partial_{t} u_{n}(x, t)=g_{n}(x, t) \text { for } x \in \Omega_{l} \cup \Omega_{r} \\
u_{n}(0, t)=0 \\
u_{n}(L, t)=0 \\
\partial_{x} u_{n}\left(d^{-}, t\right)=\partial_{x} u_{n}\left(d^{+}, t\right)=\partial_{x} u_{n}(d, t) \\
F_{0} \partial_{x} u_{n}(d, t)-K_{1} u_{n}\left(d^{+}, t\right)+K_{1} u_{n}\left(d^{-}, t\right)=r_{n}(t)
\end{array}\right.
$$

with the "input force" $g_{n}$ given by

$$
g_{1}(x, t)=\frac{1}{F_{0}} f(x, t)
$$


and, for $n \geq 2$,

$$
\begin{aligned}
g_{n}(x, t)= & \epsilon \sum_{\substack{m \in \mathbb{N}^{2} \\
m_{1}+m_{2}=n}} \partial_{x} u_{m_{1}}(x, t) \partial_{x}^{2} u_{m_{2}}(x, t) \\
& -\beta \sum_{\substack{m \in \mathbb{N}^{3} \\
m_{1}+m_{2}+m_{3}=n}} \partial_{x} u_{m_{1}}(x, t) \partial_{x} u_{m_{2}}(x, t) \partial_{x}^{2} u_{m_{3}}(x, t),
\end{aligned}
$$

and the "residual force" $r_{n}$ at damage given by

$$
r_{1}(t)=0
$$

and, for $n \geq 2$,

$$
r_{n}(t)=\sum_{j=2}^{n} K_{j} \sum_{\substack{m \in \mathbb{N}^{j} \\ m_{1}+\cdots+m_{j}=n}} \prod_{k=1}^{j}\left(u_{m_{k}}\left(d^{+}, t\right)-u_{m_{k}}\left(d^{-}, t\right)\right) .
$$

From (10) and the expression of $g_{n}$ and $r_{n}$, we can remark that each order $u_{n}$ is solution of a linear differential problem with mixed boundary conditions, where the "input force" and part of the boundary condition are function of lower orders $u_{m}$ with $m<n$. This means that, in order to find analytical solutions for $u_{n}$, it is only needed to solve once the linear differential problem (10). Furthermore, this allows a numerical simulation of our problem, where orders $u_{n}$ will be computed iteratively.

In order to facilitate the resolution of Eq. (10), we will take the expression in the Laplace domain:

$$
\Sigma_{n}:\left\{\begin{array}{l}
\partial_{x}^{2} U_{n}(x, s)-\sigma(s) U_{n}(x, s)=G_{n}(x, s) \\
U_{n}(0, s)=0 \\
U_{n}(L, s)=0 \\
\partial_{x} U_{n}\left(d^{-}, s\right)=\partial_{x} U_{n}\left(d^{+}, s\right)=\partial_{x} U_{n}(d, s) \\
F_{0} \partial_{x} U_{n}(d, s)-K_{1} U_{n}\left(d^{+}, s\right)+K_{1} U_{n}\left(d^{-}, s\right)=R_{n}(s)
\end{array}\right.
$$

with

$$
\sigma(s)^{2}=\frac{s^{2}}{c_{L}^{2}}-\frac{\gamma s}{F_{0}},
$$

and where spatio-frequency signals $U_{n}, G_{n}$ and $R_{n}$ are the Laplace transform of spatio-temporal signal $u_{n}, g_{n}$ and $r_{n}$. 


\subsection{Analytical solution}

Let $\delta=L-d$ be the length of the right-part beam, and

$$
Q(s)=\frac{1}{K_{1} \sinh (\sigma(s) L)+F_{0} \sigma(s) \cosh (\sigma(s) \delta) \cosh (\sigma(s) d)} .
$$

Then, the solution of (15) is given by, $\forall s \neq 0$,

$$
\begin{aligned}
U_{n}(x, s)= & \frac{K_{1}}{2 \sigma(s) Q(s)} \int_{0}^{L} F_{n}(\xi, s)(\cosh (\sigma(s)(L-|x-\xi|))-\cosh (\sigma(s)(L-x-\xi))) d \xi \\
& +\frac{F_{0}}{2 Q(s)} \cosh (\sigma(s) \delta) \int_{0}^{d} F_{n}(\xi, s)(\sinh (\sigma(s)(d-|x-\xi|))-\sinh (\sigma(s)(d-x-\xi))) d \xi \\
& +\frac{\sinh (\sigma(s) x) \cosh (\sigma(s) \delta)}{Q(s)} R_{n}(s)
\end{aligned}
$$

for $x \in \Omega_{l}$, and

$$
\begin{aligned}
U_{n}(x, s)= & \frac{K_{1}}{2 \sigma(s) Q(s)} \int_{0}^{L} F_{n}(\xi, s)(\cosh (\sigma(s)(L-|x-\xi|))-\cosh (\sigma(s)(L-x-\xi))) d \xi \\
& +\frac{F_{0}}{2 Q(s)} \cosh (\sigma(s) d) \int_{d}^{L} F_{n}(\xi, s)(\sinh (\sigma(s)(\delta-|x-\xi|))-\sinh (\sigma(s)(\delta-x-\xi))) d \xi \\
& -\frac{\sinh (\sigma(s)(L-x)) \cosh (\sigma(s) d)}{Q(s)} R(s)
\end{aligned}
$$

for $x \in \Omega_{r}$. The static part, i.e. for $s=0$, is given by

$$
\begin{aligned}
U_{n}(x, 0)= & \frac{K_{1} L}{K_{1} L+F_{0}} \int_{0}^{L} F_{n}(\xi, 0)\left(\mathbb{1}_{[0, x]}(\xi) \xi+\mathbb{1}_{[x, L]}(\xi) x-\frac{\xi x}{L}\right) d \xi \\
& +\frac{F_{0}}{K_{1} L+F_{0}} \int_{0}^{d} F_{n}(\xi, 0)\left(\mathbb{1}_{[0, x]}(\xi) \xi+\mathbb{1}_{[x, d]}(\xi) x\right) \\
& +\frac{x}{F_{0}+K_{1} L} R_{n}(0)
\end{aligned}
$$


for $x \in \Omega_{l}$, and

$$
\begin{aligned}
U_{n}(x, 0)= & \frac{K_{1} L}{K_{1} L+F_{0}} \int_{0}^{L} F_{n}(\xi, 0)\left(\mathbb{1}_{[0, x]}(\xi) \xi+\mathbb{1}_{[x, L]}(\xi) x-\frac{\xi x}{L}\right) d \xi \\
& +\frac{F_{0}}{K_{1} L+F_{0}} \int_{d}^{L} F_{n}(\xi, 0)\left(L-\mathbb{1}_{[d, x]}(\xi) x-\mathbb{1}_{[x, L]}(\xi) \xi\right) d \xi \\
& -\frac{(L-x)}{F_{0}+K_{1} L} R_{n}(0)
\end{aligned}
$$

for $x \in \Omega_{r}$.

Equations (18) and (19) share the same first term, which is, to a factor, the vibration of an healthy beam of length $L$ fixed at both its boundaries. For the left part (respectively the the right part), the second term is, also to a factor, the vibration of an healthy beam of length $d$ (resp. $L-d$ ) with boundary condition fixed-free (resp. free-fixed). In both parts, the third and last term corresponds to the vibration induced by the inhomogeneous mixed boundary condition due to the damage. The same remarks can be made for the static solutions (20) and (21).

Direct numerical simulation of equations $(18-21)$ requires:

- that damping be present; if not, discretization of the frequency domain will cause leaking effects that disables the possibility to simulate transient response.

- to compute the spatial integral, which will introduce numerical approximations.

To alleviate those requirements, the next section presents a modal decomposition that can be used to solve (15).

\section{Modal decomposition}

\subsection{Determination of modal shapes}

We search for an orthonormal family of modes $\phi_{p}$ such that, $\forall p \in \mathbb{N}^{*}$, it respects

$$
\Phi: \begin{cases}\phi_{p}^{\prime \prime}(x)+\lambda^{2} \phi_{p}(x)=0 & \text { for } x \in \Omega_{l} \cup \Omega_{r} \\ \phi_{p}(0)=0 & \\ \phi_{p}(L)=0 & \\ \phi_{p}^{\prime}\left(d^{-}\right)=\phi_{p}^{\prime}\left(d^{+}\right) & \\ F_{0} \phi_{p}^{\prime}\left(d^{-}\right)-K_{1} \phi_{p}\left(d^{+}\right)+K_{1} \phi_{p}\left(d^{-}\right)=0 & \end{cases}
$$

Depending whether the damage is positioned on an anti-node or not, the mode $\phi_{p}$ can be of one of two form. 
General case: In this case, the damage is not positioned on an anti-node, i.e. $\phi_{p}^{\prime}(d) \neq 0$, and therefore the modal shape $\phi_{p}$ has a discontinuity at $x=d$. Its shape is given by

$$
\phi_{p}(x)= \begin{cases}A \sin \left(\lambda_{p} x\right) & \text { for } x \in \Omega_{l}, \\ -A \frac{\cos (\lambda d)}{\cos (\lambda \delta)} \sin \left(\lambda_{p}(L-x)\right) & \text { for } x \in \Omega\end{cases}
$$

where the wavenumber $\lambda_{p}$ is solution of the transcendental equation

$$
0=\frac{F_{0}}{K_{1}}+\frac{\operatorname{sinc}\left(\lambda_{p} d\right)}{\cos \left(\lambda_{p} d\right)}+\frac{\operatorname{sinc}\left(\lambda_{p} \delta\right)}{\cos \left(\lambda_{p} \delta\right)} .
$$

If we want the modal shape to have an unit norm, then the amplitude must be

$$
A=\sqrt{\frac{2 \cos \left(\lambda_{p} \delta\right)^{2}}{d \cos \left(\lambda_{p} \delta\right)^{2}\left(1-\operatorname{sinc}\left(2 \lambda_{p} d\right)\right)+\delta \cos (\lambda d)^{2}\left(1-\operatorname{sinc}\left(2 \lambda_{p} \delta\right)\right)}} .
$$

Particular case: In this case, the damage is exactly on an anti-node, i.e. $\phi_{p}^{\prime}(d)=0$, and the modal shape is continuous. This type of modal shape is quite rare, and appears only if there exists $(p, q) \in \mathbb{N}^{2}$ such that

$$
\frac{(p+1 / 2) \pi}{d}=\frac{(q+1 / 2) \pi}{\delta} .
$$

Its shape is then given by

$$
\phi_{p}(x)=A \sin \left(\frac{(p+1 / 2) \pi}{d} x\right) .
$$

If we want the modal shape to have an unit norm, then the amplitude must be

$$
A=\sqrt{\frac{2}{L}} .
$$

Figure 3 shows the first modal shapes for a beam with $L=1 \mathrm{~m}, F_{0}=2.1 \cdot 10^{7} \mathrm{~N}$, $d=0.7 \mathrm{~m}, K_{1}=0.7 \cdot 10^{9} \mathrm{~N} / \mathrm{m}$. All modes are quite similar in shape to those of an healthy beam with fixed boundary conditions at both its extremities, except for the discontinuity at the damage position; the fifth mode, which is a particular case where the damage position corresponds exactly to the fourth anti-node, is equal to the corresponding healthy mode. 


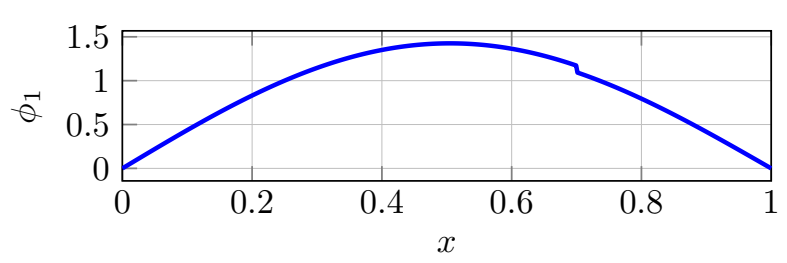

(a) Mode 1

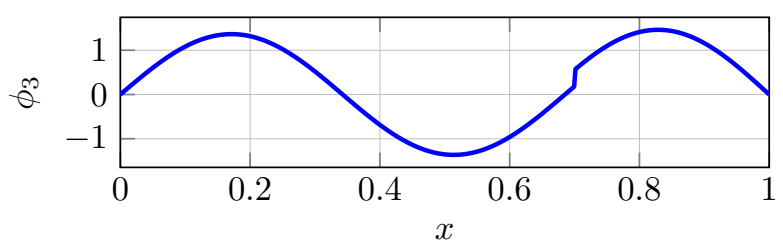

(c) Mode 3

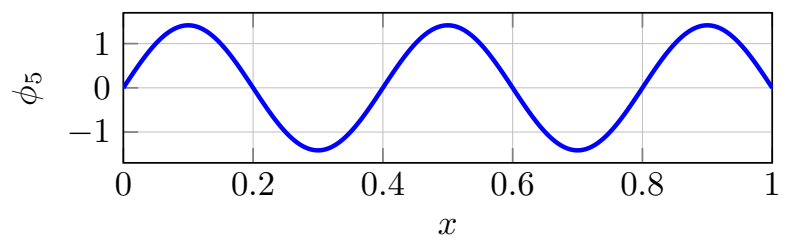

(e) Mode 5

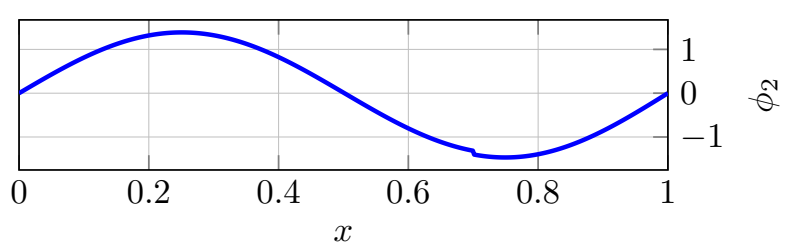

(b) Mode 2

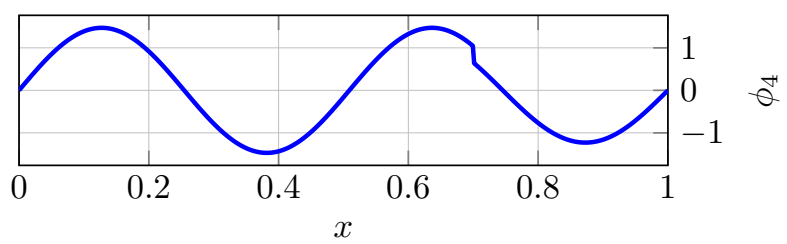

(d) Mode 4

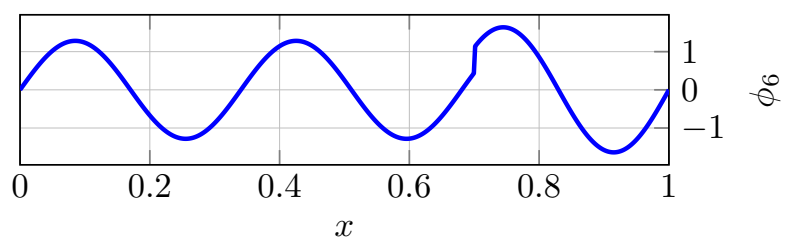

(f) Mode 6

Fig. 3: The first six modal shapes for a damaged beam (with $L=1 \mathrm{~m}, F_{0}=$ $2.1 \cdot 10^{7} \mathrm{~N}, d=0.7 \mathrm{~m}$ and $K_{1}=0.7 \cdot 10^{9} \mathrm{~N} / \mathrm{m}$ ); the fifth one is a particular case, where the damage position corresponds to the fourth anti-node.

\subsection{Modal solution}

Once the modal decomposition for the damaged beam is computed, it is possible to solve (15) if the assumption that the solution $U_{n}$ can be decomposed on a modal basis is made. Because problem (15) contains one inhomogeneous boundary condition (i.e. $F_{0} \partial_{x} U_{n}(d, s)-K_{1} U_{n}\left(d^{+}, s\right)+K_{1} U_{n}\left(d^{-}, s\right)=R_{n}(s)$ ), we also need to add a particular solution that takes it into account. Therefore the modal solution of problem (15) is given by

$$
U_{n}(x, s)=h(x) R_{n}(s)+\sum_{p=1}^{+\infty} \phi_{p}(x) U_{n, p}(s)
$$


where $h$ is a spatial shape with null second derivative that respects condition $F_{0} \partial_{x} h(d)-$ $K_{1} h\left(d^{+}\right)+K_{1} h\left(d^{-}\right)=1$, given by

$$
h(x)= \begin{cases}\frac{x}{F_{0}+K_{1} L} & \text { for } x \in \Omega_{l}, \\ -\frac{(L-x)}{F_{0}+K_{1} L} & \text { for } x \in \Omega_{r},\end{cases}
$$

and $U_{n, p}$ is the spectral response of the $p$-th mode of $U_{n}$, given by the equation

$s^{2} U_{n, p}(s)+\frac{\gamma s}{\rho S} U_{n, p}(s)+\lambda^{2} c_{L}^{2} U_{n, p}(s)=-c_{L}^{2}\left\langle G_{n}, \phi_{p}\right\rangle(s)+\left\langle h, \phi_{p}\right\rangle\left(\frac{\gamma s}{\rho S} R_{n}(s)-R_{n}(s)\right)$,

or equivalently the ordinary differential equation

$$
\ddot{u}_{n, p}(t)+\frac{\gamma}{\rho S} \dot{u}_{n, p}(t)+\lambda^{2} c_{L}^{2} u_{n, p}(t)=-c_{L}^{2}\left\langle g_{n}, \phi_{p}\right\rangle(t)+\left\langle h, \phi_{p}\right\rangle\left(\frac{\gamma}{\rho S} \dot{r}_{n}(t)-\ddot{r}_{n}(t)\right),
$$

where the notation $\langle a, b\rangle$ corresponds to the spatial scalar product between $a$ and $b$, i.e.

$$
\langle a, b\rangle=\int_{0}^{L} a(x) b(x) d x .
$$

The modal solution of (15) can thus be numerically simulated, for each order $n$, as follows:

1. compute terms $g_{n}$ and $r_{n}$ from previous orders $u_{m}, m<n$; for $n=1$, we have $g_{1}(x, t)=f(x, t)$ and $r_{n}(t)=0$;

2. numerically solve (32) using a discretization method (Euler method, bilinear transform, first-order hold, etc);

3. compute the solution using (29).

\section{Simulation and comparison with state-of-the-art}

In this section, we will compare numerical simulations of the two solutions of the damaged beam problem (7) (given in Sections 3.3 and 4.2) with SDTools Matlab Toolbox [10], a state-of-the-art analysis and simulation toolbox for vibration which uses finite-elements method.

\subsection{Simulation parameters}

The base model is a steel beam of length $L=1 \mathrm{~m}$, with a square section of surface $S=1 \mathrm{~cm}^{2}$, a Young modulus of $E=210.0 \mathrm{GPa}$, a density of $\rho=7850.0, \mathrm{~kg} / \mathrm{m}^{3}$, 
and a damping factor of $\gamma=5.0 \cdot 10^{3} \mathrm{kgm}^{-1} \mathrm{~s}^{-1}$. For the simulation, the propagation is supposed linear, i.e. $\epsilon=0$ and $\beta=0$.

The damage is taken as a cubic spring, i.e. with a characteristic relation given by

$$
K[x]=K_{1} x+K_{3} x^{3},
$$

where $K_{1}=0.7 \mathrm{e} \cdot 10^{9} \mathrm{~N} / \mathrm{m}$ and $K_{3}=5.0 \cdot 10^{27} \mathrm{~N} / \mathrm{m}^{3}$. It is placed at $d=0.7 \mathrm{~m}$.

The input force is taken as sine burst excitation, located at $x_{0}=0.3 \mathrm{~m}$. It is comprised of three cycles at $f_{0}=100 \mathrm{kHz}$ multiplied by a Hann window of unit amplitude, sampled at $f_{s}=5 \mathrm{MHz}$. Output vibration is simulated for a duration of $1 \mathrm{~ms}$.

For both simulation methods presented in this paper, only the $N=15$ first orders of the series are simulated. The analytical solution simulation uses a zero-padding of 50000 points to avoid leaking effects due to windowing. The modal solution simulation uses a number of 100 modes and a first-order hold as discretization method.

\subsection{Simulation results}

Figure 4 shows the results for the three simulation. We can see that obtained results are qualitatively similar for all three simulations, with small differences in the damping of the vibrations. The damage acts as a nonlinear semi-reflecting barrier, which creates harmonic distortion (visible in the output spectra).

Furthermore, we can quantitatively compare simulations by computing the RMS value of their difference, which gives:

- a value of $-14 \mathrm{~dB}$ between the analytical approach and the result given by SDTools;

- a value of $-13 \mathrm{~dB}$ between the modal approach and the result given by SDTools;

- a value of $-16 \mathrm{~dB}$ between the modal and analytical approach.

Therefore, the simulations using both proposed approaches and through SDTools obtain qualitatively and quantitatively similar results, thus validating the approaches. 


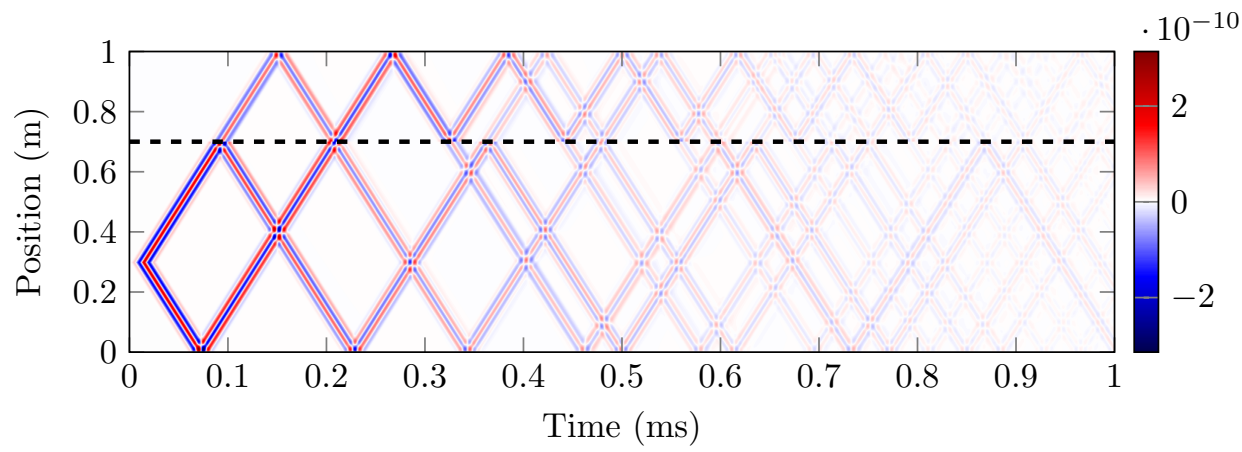

(a) Analytical solution

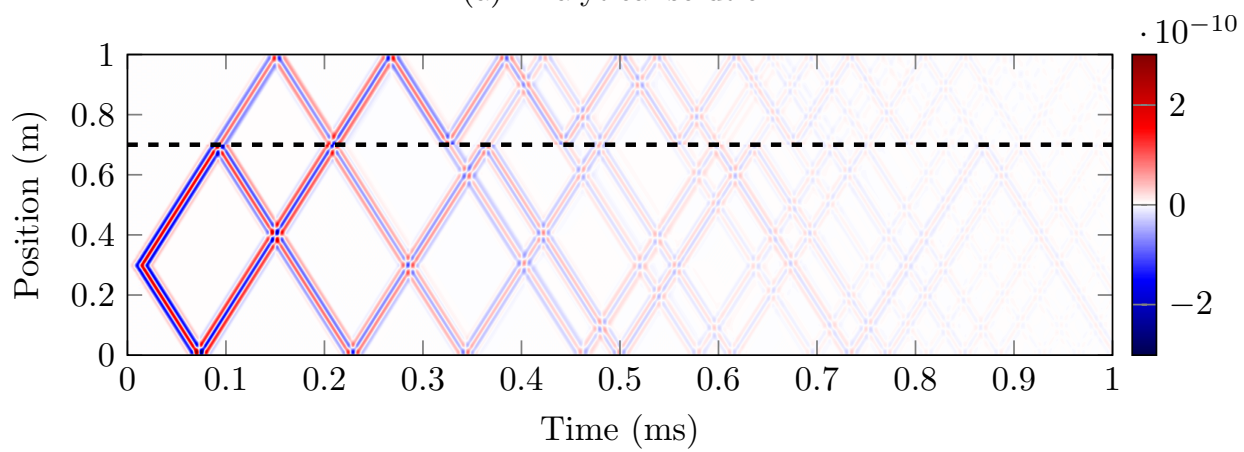

(b) Modal solution

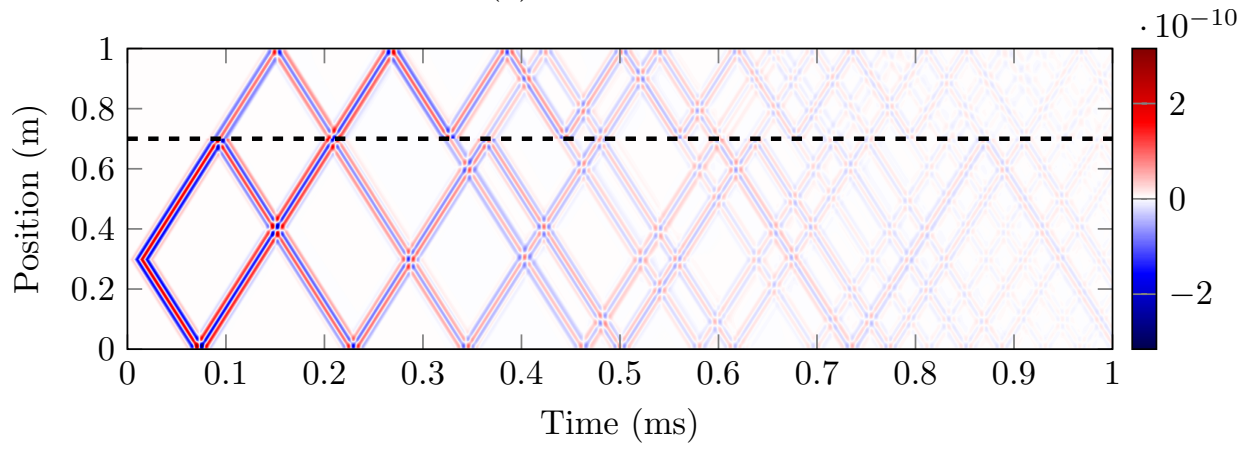

(c) SDTools toolbox

Fig. 4: Simulations of the damaged beam using (a) the analytical solution (see Section 3.3), (b) the modal solution (see Section 4.2), (c) a state-of-the-art vibration toolbox avalaible for Matlab; simulations parameters are presented in Section 5.1. 


\section{Conclusion}

In this study, we have shown how the Green-Volterra formalism can be used to find analytical solutions for the S0 mode nonlinear propagation and nonlinear damage interaction in a damaged beam. Furthermore, those solutions can be decomposed onto an appropriate modal basis for simulation purposes. Finally, we have shown through a simulation example that both proposed approach for simulations gave qualitatively and quantitatively similar results with a state-of-the-art finite element method.

Future research will focus on using the obtained analytical model to derive SHM tools for damage classification or quantification, as well as extending the proposed approach to other types of vibration, e.g. transverse A0 mode or other Lamb wave modes.

\section{Acknowledgements}

This work has received funding from the European Union's Horizon 2020 research and innovation program under the REMAP project (grant agreement number 769288).

\section{References}

1. Rytter, A.: Vibrational Based Inspection of Civil Engineering Structures. Ph.D. thesis, Denmark (1993)

2. Worden, K., Farrar, C.R., Haywood, J., Todd, M.: A review of nonlinear dynamics applications to structural health monitoring. Structural Control and Health Monitoring 15(4), 540-567 (2008)

3. Rébillat, M., Hajrya, R., Mechbal, N.: Nonlinear structural damage detection based on cascade of hammerstein models. Mechanical Systems and Signal Processing 48(1-2), 247-259 (2014)

4. Ghrib, M., Rébillat, M., des Roches, G.V., Mechbal, N.: Automatic damage type classification and severity quantification using signal based and nonlinear model based damage sensitive features. Journal of Process Control (2018)

5. Rugh, W.J.: Nonlinear system theory. Johns Hopkins University Press Baltimore (1981)

6. Boyd, S., Chua, L.O.: Fading memory and the problem of approximating nonlinear operators with Volterra series. IEEE Transactions on Circuits and Systems 32(11), 1150-1161 (1985)

7. Li, H.X., Qi, C., Yu, Y.: A spatio-temporal volterra modeling approach for a class of distributed industrial processes. Journal of Process Control 19(7), 1126-1142 (2009)

8. Roze, D., Hélie, T.: Introducing a green-volterra series formalism to solve weakly nonlinear boundary problems: Application to kirchhoff's string. Journal of Sound and Vibration 333(7), 2073-2086 (2014) 
9. Broda, D., Staszewski, W., Martowicz, A., Uhl, T., Silberschmidt, V.: Modelling of nonlinear crack-wave interactions for damage detection based on ultrasound - a review. Journal of Sound and Vibration 333(4), 1097-1118 (2014)

10. Balmès, E.: Sdtools, vibration software and consulting (2020), https://www.sdtools.com/ 